\title{
Efectos de la gestión forestal en los flujos de nutrientes asociados al ciclo hidrológico en un bosque mediterráneo de Quercus Ilex
}

\author{
Bautista I. ${ }^{*}$, Pabón C. ${ }^{2}$, Lull C. ${ }^{1}$, González-Sanchís M. ${ }^{1}$, Lidón A. ${ }^{1}$, del Campo A. ${ }^{1}$ \\ ${ }^{1}$ Grupo ReForest, Departamento de Ingeniería Hidráulica y Medio Ambiente, Universitat Politècnica de València, \\ Camino de Vera s/n, 46022, Valencia. España. \\ ${ }^{2}$ Universidad Francisco de Paula Santander, Barrio Colsag, Cúcuta, Norte de Santander, Colombia. \\ *e-mail: ibautista@qim.upv.es
}

\section{Resumen}

Las interacciones entre cubierta forestal y agua son complejas ya que afectan a diferentes variables tales como el reparto de los flujos de agua, la recolección de agua y la calidad de la misma. El crecimiento forestal en suelos de baja fertilidad requiere que los ciclos de nutrientes dentro del ecosistema sean eficientes, especialmente en bosques mediterráneos en los que, además de los problemas de escasez de agua, el nitrógeno y el fósforo son a menudo nutrientes limitantes. El objetivo de este trabajo es analizar el impacto de la gestión forestal sobre los flujos de nutrientes (C, N, P y K) asociados al ciclo hidrológico. Se seleccionó un bosque de encina situado en el monte público de La Hunde, en la provincia de Valencia. Se establecieron dos parcelas contiguas, control y tratamiento, de $1800 \mathrm{~m}^{2}$ cada una. En la parcela tratamiento se redujo la densidad de 861 a 414 árboles por ha, siguiendo los requisitos del gestor forestal. Se han medido los flujos de trascolación, escorrentía cortical y se han instalado zanjas de drenaje para recoger la escorrentía superficial y subsuperficial. Durante más de un año hidrológico se han recogido muestras de agua en ambas parcelas, con una periodicidad aproximadamente quincenal, y se han medido los contenidos de C, N, P y K disueltos en el agua, tanto en forma orgánica como inorgánica, para analizar los efectos del tratamiento en el ciclo de nutrientes.

Palabras clave: ciclo de nutrientes, escorrentía, escorrentía cortical, gestión forestal, trascolación. 


\section{Introducción}

Los ecosistemas mediterráneos se consideran pobres en nutrientes. Los suelos mediterráneos son suelos jóvenes y poco profundos debido a la escasez de agua y están sujetos a un alto grado de erosión debido a la irregularidad de las precipitaciones (Yaalon, 1997). Aparte de las restricciones hídricas, los dos nutrientes que más limitan el crecimiento en los ecosistemas mediterráneos son el $\mathrm{N}$ y el $\mathrm{P}$, aunque con un comportamiento bastante diferenciado. E1 N presenta formas gaseosas y su mayor masa se encuentra en la atmósfera, siendo la fuente primaria de entrada a los ecosistemas las entradas atmosféricas, bien directamente a través de deposición, o bien mediante colonización del medio por especies fijadoras de nitrógeno cuyos residuos van acumulándose en la materia orgánica del suelo. En cambio el $\mathrm{P}$, a pesar de que puede entrar en el sistema a través de deposiciones sólidas, no dispone de formas gaseosas; su principal fuente en el ecosistema es a través de la meteorización de minerales que contienen fósforo (Sardans et al., 2004).

En ecosistemas pobres en nutrientes los árboles desarrollan sus ciclos biológicos gracias a su capacidad para conservar y reciclar los nutrientes. En ecosistemas mediterráneos predominan especies esclerófilas con hojas de elevada longevidad donde la reabsorción supone un importante mecanismo de conservación de nutrientes, en especial en el caso del N y P. Este proceso consiste en la hidrólisis de los nutrientes contenidos en la hoja senescente y el transporte de los productos resultantes a tejidos en crecimiento o hacia zonas perennes de la planta donde son almacenados hasta su posterior utilización (Thomas y Stoddart, 1980).

Los aportes al suelo por parte de la planta se basan en el desfronde y el lavado (Kimmins, 1997). El retorno de materia orgánica y elementos minerales a través de la caída de las partes aéreas de la planta (desfronde) junto con la tasa de renovación de la biomasa subterránea constituye el principal proceso de transferencia de nutrientes al suelo (Vitousek et al., 1995). El reciclado de los nutrientes aportados por el desfronde puede llegar a suponer más del 90\% del nitrógeno y fósforo absorbido por las plantas (Chapin et al., 2002), por lo que juega un papel fundamental en el ciclo de nutrientes y en la transferencia de energía entre las plantas y el suelo.

El agua de la lluvia que cae sobre la vegetación arrastra los nutrientes depositados sobre la superficie de las hojas o los que puede disolver de las hojas y el tronco, transportándolos hasta el suelo a través de la copa (trascolación) o a través de las ramas y el tronco (escorrentía cortical). El aporte de nutrientes constituye en promedio el 15\% del retorno anual (Chapin et al., 2002), pero es variable y depende del elemento considerado.

En trabajos previos se ha analizado principalmente el impacto de la gestión del bosque sobre los flujos de nutrientes en forma inorgánica. Sobre todo se ha encontrado un incremento de las pérdidas de nitrógeno en forma nítrica tras una tala debido a la disminución de absorción radicular (Bormann y Likens, 1979) o al incremento del flujo de agua como consecuencia de la reducción de la transpiración (Likens y Bormann, 1995). Otros estudios más recientes señalan que la materia orgánica disuelta constituye el principal medio para el transporte y la pérdida de $\mathrm{N}$ y $\mathrm{P}$ en los eco- 
sistemas forestales (Qualls y Haines, 1991; Perakis y Hedin, 2002). Algunos autores indican un patrón inverso entre las concentraciones de nitrato y nitrógeno orgánico disuelto (NOD) en la escorrentía, con concentraciones más altas de nitrato al final del invierno y concentraciones más altas de NOD en verano y comienzo de otoño (Vanderbilt et al., 2003). Qualls y Haines (1992) sugieren que la abundancia de NOD se debe a que los microorganismos no lo degradan debido a que se encuentra en forma de ácidos fúlvicos procedentes de la materia orgánica estable del suelo. En ambientes mediterráneos se ha encontrado que los nutrientes transportados por los cursos de agua durante los meses que siguen el periodo seco del verano son muy diferentes a los del resto del año (Ávila et al., 1992, Bernal et al. 2005). Este efecto se explica en parte por los pulsos de descomposición de la materia orgánica, asociados a incrementos bruscos de humedad, que se producen en suelos forestales tras un periodo seco (Lado-Monserrat et al., 2014).

Hasta el momento no se ha analizado de forma directa el efecto de los tratamientos selvícolas sobre la concentración de nutrientes en flujos de agua en bosques mediterráneos. Nuestro objetivo es analizar el impacto de la gestión forestal sobre los flujos de nutrientes (C, N, P y K) asociados al ciclo hidrológico y comparar las concentraciones de nutrientes disueltos en forma orgánica y mineral: nitrógeno orgánico disuelto vs nitrógeno en las formas nítrica y amoniacal y fósforo orgánico disuelto vs fósforo en forma de fosfato.

\section{Material y métodos}

En un bosque de encina situado en el monte público de La Hunde, Valencia (NE España), se establecieron dos parcelas contiguas en la ladera noroeste, control (C) y tratamiento (T), de $1800 \mathrm{~m}^{2}$ cada una. En la parcela de tratamiento se reduce la densidad de 861 a 414 árboles por ha, siguiendo los criterios del gestor forestal. La orientación de la ladera es NNW, la altitud es de $962 \mathrm{~m}$ snm y la pendiente media es de $15^{\circ}$. En la parte alta de la pendiente aparecen afloramientos rocosos, con rodales cubiertos de musgo, donde prolifera la especie Juniperus faenicia. En la fecha del tratamiento, mayo de 2012, se realizó un muestreo de suelo en 4 puntos repartidos a lo largo de la pendiente en cada una de las parcelas.

En noviembre de 2012 se excavaron dos zanjas de drenaje de $4 \mathrm{~m}$ de longitud en la parte inferior de cada una de las parcelas, perpendicularmente a la pendiente, para recoger la escorrentía superficial y subsuperficial. En febrero de 2013 se instalaron colectores de $1 \mathrm{~m}^{2}$, en distintos puntos de la pendiente, conectados a pluviómetros de cuchara para medir la trascolación. También se instalaron varios colectores similares fuera de la masa forestal para medir la precipitación. Los árboles de ambas parcelas se clasificaron en cuatro clases diamétricas, y en cada una de éstas se seleccionó un árbol por parcela para recoger la escorrentía cortical mediante embudos situados en la parte baja del tronco. Los embudos drenaban hacia depósitos donde se almacena el agua de escorrentía, midiéndose el volumen manualmente. Durante más de un año se han venido recogiendo muestras de agua en ambas parcelas, con una periodicidad 
aproximadamente quincenal, para analizar los contenidos de C, N, P y K disueltos en el agua, tanto en forma orgánica como inorgánica. Las muestras eran transportadas al laboratorio donde se congelaban hasta el momento de su análisis. Las muestras se filtraron a través de papel de filtro Whatman $\mathrm{n}^{\circ}{ }^{\mathrm{a}} 42$, para obtener los nutrientes solubles analizándose potasio, amonio, nitrato, fosfato, carbono orgánico disuelto (COD), nitrógeno orgánico disuelto (NOD) y fósforo orgánico disuelto (POD).

El potasio en disolución se determinó por fotometría de llama en fotómetro Jenway PFP7. Para la determinación del nitrógeno en forma nítrica y amónica se empleó el método FIA (análisis por inyección de flujo). El principio químico de la determinación de nitrato es la reducción de éste a nitrito en una columna de $\mathrm{Cd}$ y posterior determinación colorimétrica con N-(1-Naftil)-etilendiaminadiclorhidrato y sulfanilamida. Se mide la absorbancia de la mezcla obtenida a $540 \mathrm{~nm}$ (Tecator Aplication Note AN 5201, 1984). La determinación del amonio se basa en la determinación colorimétrica del amonio liberado tras reaccionar la muestra de agua con hidróxido sódico. El amoniaco gaseoso se difunde a través de una membrana permeable dentro de una mezcla de indicadores ácido-base y se mide la absorbancia de la mezcla a $590 \mathrm{~nm}$ (Tecator Aplication Note AN 5226, 1984). Para la determinación de fósforo se utiliza el método del ácido ascórbico (Kuo, 1996).

El COD se analizó midiendo la absorbancia del Cr en forma oxidada por el método de Yakovchenko y Sikora (1998). A $2 \mathrm{~mL}$ de la muestra de agua se añadieron 3 $\mathrm{mL}$ de $\mathrm{K}_{2} \mathrm{Cr}_{2} \mathrm{O}_{7} 0.20 \mathrm{~N}$ en $\mathrm{H}_{2} \mathrm{SO}_{4} 26.7 \mathrm{~N}$, posteriormente se pusieron en estufa a 140 ${ }^{\circ} \mathrm{C}$ durante 20 minutos y seguidamente se leyó la absorbancia a una longitud de onda de $590 \mathrm{~nm}$. El N y P disueltos totales se determinaron mediante digestión con persulfato potásico a $121{ }^{\circ} \mathrm{C}$ durante 1 hora (Patton and Kryskalla, 2003) y posteriormente mediante el análisis de nitrógeno nítrico y fósforo en forma de fosfato por los métodos anteriormente citados en los digestos, ya que en el proceso de digestión todas las formas de $\mathrm{N}$ pasan a nitrato y todas las formas de $\mathrm{P}$ a fosfato. El NOD se obtiene por diferencia entre el $\mathrm{N}$ disuelto total menos la suma de nitrógeno en forma nítrica y amónica. El POD se calcula como el $\mathrm{P}$ disuelto total menos el $\mathrm{P}$ inorgánico. También se ha medido la absorbancia en UV a $280 \mathrm{~nm}$ en cubeta de cuarzo en un espectrofotómetro UNICAM UV/Vis (Chantigny et al. 2008).

Para el análisis estadístico de los datos se ha utilizado el programa Statgraphics Centurion XVI. Se ha aplicado análisis de la varianza (ANOVA) múltiple sin interacción para analizar el efecto de la fecha de muestreo y el origen del agua sobre las concentraciones de los nutrientes. Se ha utilizado el coeficiente de correlación de Pearson para ver las relaciones significativas entre las formas químicas y el volumen de agua recogido, separadamente en los diferentes tipos de flujo.

\section{Resultados y discusión}

\subsection{Características de los suelos de las parcelas}

Los suelos de las parcelas son poco profundos; en la parte inferior de la ladera 
alcanzan hasta $50 \mathrm{~cm}$ de profundidad pero, en algunas zonas de la parte alta, la capa de mantillo se encuentra directamente sobre la roca madre. El horizonte superficial del suelo presenta una textura franca con un $44 \%$ de arena, $33 \%$ limo y $23 \%$ de arcilla. La pedregosidad es muy alta (alrededor del 50\%), incluso en la capa de hojarasca, y aumenta ligeramente con la profundidad (Tab. 1). El pH de los suelos es básico y oscila entre 7.84 en la capa de mantillo y 8.34 a $30 \mathrm{~cm}$ de profundidad. Los suelos presentan concentraciones apreciables de $\mathrm{CaCO}_{3}$ que oscilan entre $15 \%$ en la capa de mantillo y $37 \%$ a profundidad superior a $30 \mathrm{~cm}$. El contenido de $\mathrm{C}$ orgánico oxidable varía en profundidad en proporción inversa a la del C inorgánico, presentando un valor máximo en la capa de mantillo que desciende a $25 \mathrm{~g} \mathrm{~kg}^{-1}$ a profundidades superiores a $30 \mathrm{~cm}$ (Tab. 1).

Tabla 1. Valores medios (Media) y desviación estándar ( $\mathrm{Sd}$ ) de las propiedades de los suelos en función de la profundidad ( $\mathrm{n}=8$ puntos).

\begin{tabular}{|l|c|c|c|c|c|c|c|c|}
\hline \multirow{2}{*}{ Capa } & \multicolumn{2}{|c|}{$\begin{array}{c}\text { Pedregosidad } \\
\%\end{array}$} & \multicolumn{2}{c|}{$\mathrm{pH}$} & \multicolumn{2}{c|}{$\begin{array}{c}\mathrm{CaCO}_{3} \\
\%\end{array}$} & \multicolumn{2}{c|}{$\begin{array}{c}\text { Corg oxidable } \\
\mathrm{g} \mathrm{kg}^{-1}\end{array}$} \\
\cline { 2 - 9 } & Media & $\mathrm{Sd}$ & Media & $\mathrm{Sd}$ & Media & $\mathrm{Sd}$ & Media & $\mathrm{Sd}$ \\
\hline Hojarasca & 48.4 & 10.7 & & & & & & \\
Mantillo & 59.2 & 7.1 & 7.84 & 0.09 & 15.3 & 5.6 & 131.2 & 32.0 \\
Suelo 0-10 cm & 63.9 & 8.5 & 8.05 & 0.11 & 21.1 & 6.7 & 73.2 & 17.4 \\
Suelo 10-30 cm & 58.6 & 7.3 & 8.25 & 0.12 & 34.1 & 6.2 & 42.3 & 21.4 \\
Suelo 30-50 cm & 55.5 & 7.2 & 8.34 & 0.04 & 36.7 & 1.7 & 25.1 & 6.4 \\
\hline
\end{tabular}

\subsection{Distribución de la precipitación}

La precipitación del periodo estudiado entre el 14/11/2012 y el 18/02/2014 es de 678. $1 \mathrm{~mm}$ (Tab. 2), está bien distribuida a los largo del año y sin periodos de sequía de larga duración (Fig. 1). Las precipitaciones más bajas se localizan en los meses de verano, situación normal en el clima mediterráneo. Teniendo en cuenta la precipitación anual media de la zona, alrededor de $450 \mathrm{~mm}$, se puede considerar el periodo analizado como bastante húmedo.

La escorrentía superficial es extremadamente baja (Tab. 2) a pesar de la pendiente, $\mathrm{y}$ representa un porcentaje similar de la precipitación en ambas parcelas ( $\mathrm{T}$ y $\mathrm{C}$ ). Al ser los suelos de las parcelas muy pedregosos y de textura franca, la infiltración es muy alta y no hay pérdidas altas por escorrentía superficial o subsuperficial. La trascolación representa el $52 \%$ de la precipitación en la parcela control mientras que en la parcela tratada asciende al 62\%. La parcela control, al presentar mayor cubierta arbórea, intercepta un porcentaje mayor de la precipitación. La escorrentía cortical es bastante baja (0.6-0.7\% de la precipitación) y tiene un valor ligeramente más alto en la parcela control. Estos valores contrastan con los valores medios de 4.2\% presentados por Llorens y Domingo (2007) para Quercus ilex, posiblemente debi- 


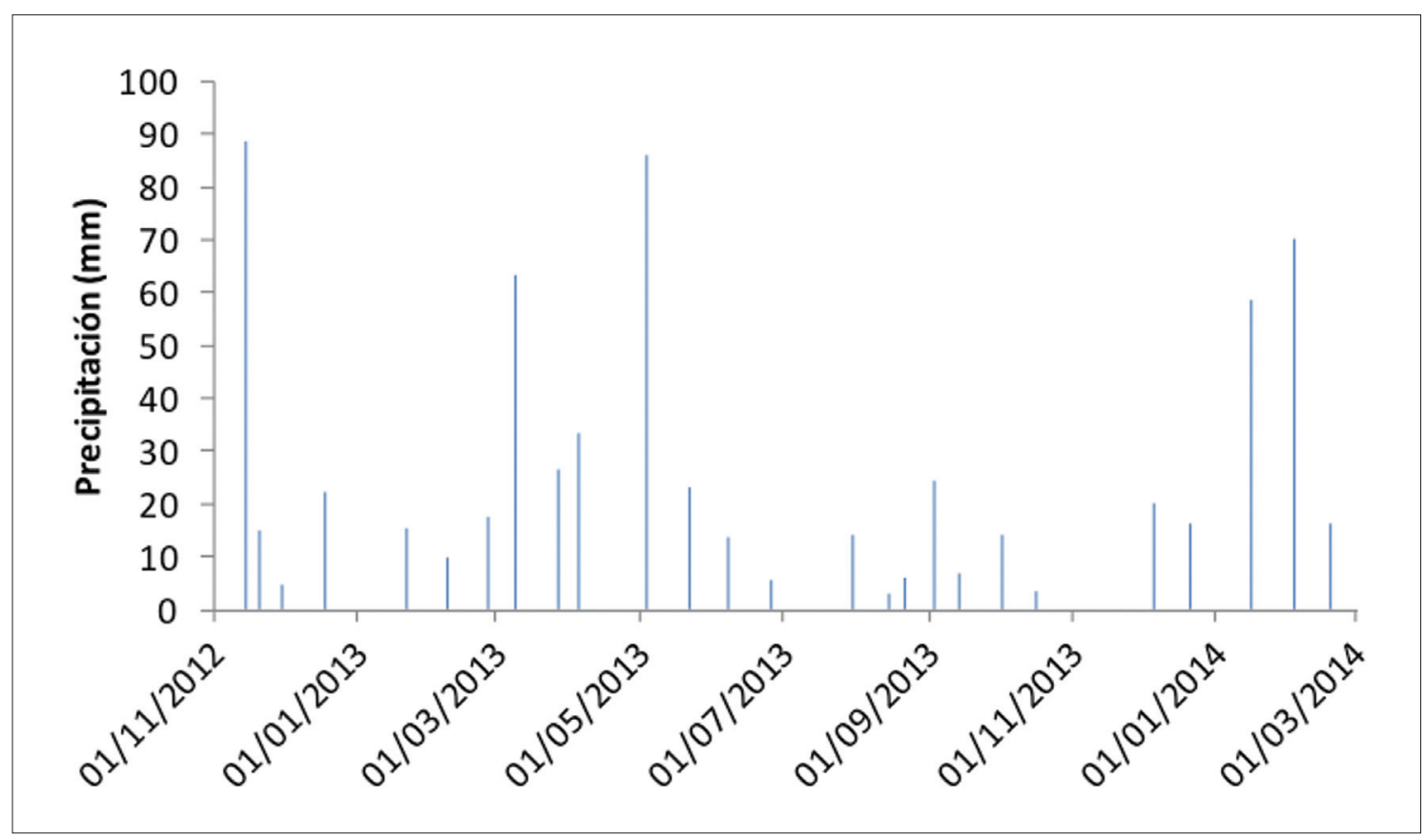

Figura 1. Distribución de la precipitación en las parcelas experimentales, medida en intervalos de quince días.

do a la alta variabilidad en la estructura espacial de la parte aérea entre árboles de la misma especie.

Tabla 2. Distribución de la lluvia en la parcela control (C) y tratada (T). Todos los volúmenes están expresados en $\mathrm{mm}$.

\begin{tabular}{|c|c|c|c|c|c|c|c|}
\hline \multirow[t]{2}{*}{ Periodo } & \multirow[t]{2}{*}{ Lluvia } & \multicolumn{2}{|c|}{ Trascolación } & \multicolumn{2}{|c|}{$\begin{array}{l}\text { Escorrentía } \\
\text { cortical }\end{array}$} & \multicolumn{2}{|c|}{$\begin{array}{l}\text { Escorrentía } \\
\text { subsuperficial }\end{array}$} \\
\hline & & $\mathrm{C}$ & $\mathrm{T}$ & $\mathrm{C}$ & $\mathrm{T}$ & $\mathrm{C}$ & $\mathrm{T}$ \\
\hline $14 / 11 / 2012$ a $21 / 01 / 2013$ & 145.8 & & & & & 0.25 & 0.33 \\
\hline $21 / 01 / 2013$ a $18 / 02 / 2014$ & 532.3 & 276.0 & 330.2 & 3.69 & 3.02 & 2.11 & 2.08 \\
\hline
\end{tabular}

\subsection{Concentraciones de nutrientes en el agua}

Las medidas de nutrientes disueltos en los distintos flujos de agua son extremadamente variables (presentan valores de desviaciones estándar del mismo orden de magnitud que los valores medios). El ANOVA múltiple aplicado sin interacciones (Tab. 3) indica que la mayoría de las variables difieren significativamente en función de la procedencia del agua. En general, los valores más altos de concentración se presentan en la escorrentía cortical y los valores más bajos en la trascolación. En el caso de la absorbancia en ultravioleta, carbono orgánico disuelto y nitrógeno orgánico di- 
suelto se presentan diferencias significativas entre fechas. En ningún caso se producen diferencias significativas entre tratamientos selvícolas.

Tabla 3. Valores medios y desviaciones estándar de lámina de agua ( $\mathrm{mm})$, absorbancia a $280 \mathrm{~nm}$ (Abs UV) y las concentraciones de COD (carbono orgánico disuelto), POD (fósforo orgánico disuelto), $\mathrm{P}_{-} \mathrm{PO}_{4}$ (fósforo inorgánico), $\mathrm{NOD}$ (nitrógeno orgánico disuelto), $\mathrm{N}-\mathrm{NO}_{3}$ (nitrógeno en forma nítrica) $\mathrm{N}_{-} \mathrm{NH}_{4}$ (nitrógeno en forma amoniacal) y K (potasio) en las parcelas control (C) y tratada (T)

\begin{tabular}{|c|c|c|c|c|c|c|c|}
\hline \multirow{2}{*}{$\begin{array}{l}\text { Variable } \\
\text { Parcela }\end{array}$} & \multicolumn{2}{|c|}{ Escorrentía suelo } & \multicolumn{2}{|c|}{ Escorrentía cortical } & \multicolumn{2}{|c|}{ Trascolación } & \multirow{2}{*}{$\begin{array}{l}\text { Efectos } \\
\text { significativo }\end{array}$} \\
\hline & $\mathrm{C}$ & $\mathrm{T}$ & $\mathrm{C}$ & $\mathrm{T}$ & $\mathrm{C}$ & $\mathrm{T}$ & \\
\hline Lámina mm & $0.11 \pm 0.16$ & $0.12 \pm 0.18$ & $0.41 \pm 0.37$ & $0.27 \pm 0.22$ & $15.34 \pm 32.59$ & $18.38 \pm 29.4$ & Origen \\
\hline $\mathrm{COD} \mathrm{mg} \mathrm{L}^{-1}$ & $22.7 \pm 17.3$ & $37.2 \pm 24.9$ & $52.6 \pm 25.4$ & $60.3 \pm 27.4$ & $27.5 \pm 31.6$ & $22.4 \pm 26.5$ & Origen.Fecha \\
\hline Abs UV & $952 \pm 601$ & $1360 \pm 574$ & $2118 \pm 1516$ & $1862 \pm 520$ & $373 \pm 271$ & $233 \pm 237$ & Origen.Fecha \\
\hline POD mg L-1 & $0.11 \pm 0.25$ & $0.07 \pm 0.03$ & $0.24 \pm 0.79$ & $0.03 \pm 0.23$ & $0.02 \pm 0.03$ & $0.01 \pm 0.01$ & NS \\
\hline $\mathrm{P}_{-} \mathrm{PO}_{4} \mathrm{mg} \mathrm{L}^{-1}$ & $0.12 \pm 0.23$ & $0.03 \pm 0.06$ & $0.84 \pm 1.79$ & $0.56 \pm 1.18$ & $0.00 \pm 0.00$ & $0.00 \pm 0.00$ & Origen \\
\hline NOD mg L ${ }^{-1}$ & $2.63 \pm 1.03$ & $3.20 \pm 1.24$ & $2.78 \pm 1.88$ & $1.92 \pm 3.06$ & $1.12 \pm 1.26$ & $0.73 \pm 1.07$ & Origen.Fecha \\
\hline 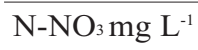 & $2.10 \pm 1.23$ & $1.36 \pm 1.27$ & $1.38 \pm 3.83$ & $3.49 \pm 9.26$ & $0.70 \pm 0.60$ & $0.66 \pm 0.63$ & NS \\
\hline $\mathrm{N}^{-\mathrm{NH}_{4}} \mathrm{mg} \mathrm{L}^{-1}$ & $0.98 \pm 1.91$ & $0.19 \pm 0.24$ & $1.87 \pm 5.00$ & $15.55 \pm 13.29$ & $0.16 \pm 0.23$ & $0.11 \pm 0.09$ & Origen \\
\hline $\mathrm{K} \mathrm{mg} \mathrm{L}^{-1}$ & $6.93 \pm 4.90$ & $16.54 \pm 7.43$ & $24.37 \pm 21.34$ & $15.29 \pm 7.38$ & $4.23 \pm 3.60$ & $3.12 \pm 2.89$ & Origen \\
\hline
\end{tabular}

Si se analiza la dinámica temporal del COD, las concentraciones más altas en la escorrentía del suelo se concentran en los meses posteriores a la sequía estival (Fig 2.1), y normalmente presentan valores más altos en la parcela tratada. En el caso de la escorrentía cortical (Fig 2.2), los valores son más constantes a lo largo del año aunque también se aprecia un descenso de concentración en los meses de invierno. No en todos los eventos de lluvia se produce escorrentía cortical, ya que sólo se recoge con precipitaciones superiores a $10 \mathrm{~mm}$. La tendencia entre parcelas varía temporalmente: en los meses de verano y otoño la concentración de COD es mayor en la parcela tratada, pero en primavera se aprecian concentraciones más altas en la parcela control. Los valores de COD en las muestras de trascolación (Fig. 2.3) muestran también una tendencia estacional muy clara y valores más altos en la parcela control.

Como la concentración de nutrientes varía significativamente según el tipo de flujo de agua considerado (Tab. 3), las relaciones entre las distintas variables se analizan de forma separada en función del origen del agua (Tab. 4). Las correlaciones entre el volumen de agua recogido y las concentraciones de cada uno de los nutrientes en las muestras son, en general, negativas aunque no significativas. Cabe destacar las correlaciones positivas y altamente significativas entre el COD, la absorbancia en UV y el nitrógeno y fósforo disueltos en forma orgánica.

El fosforo inorgánico se correlaciona positivamente con el catión amonio cuando se consideran los flujos de agua a través del suelo (escorrentía). Cuando se analiza la escorrentía cortical, este anión se correlaciona principalmente con el K pero 


\section{Escorrentía en el suelo}

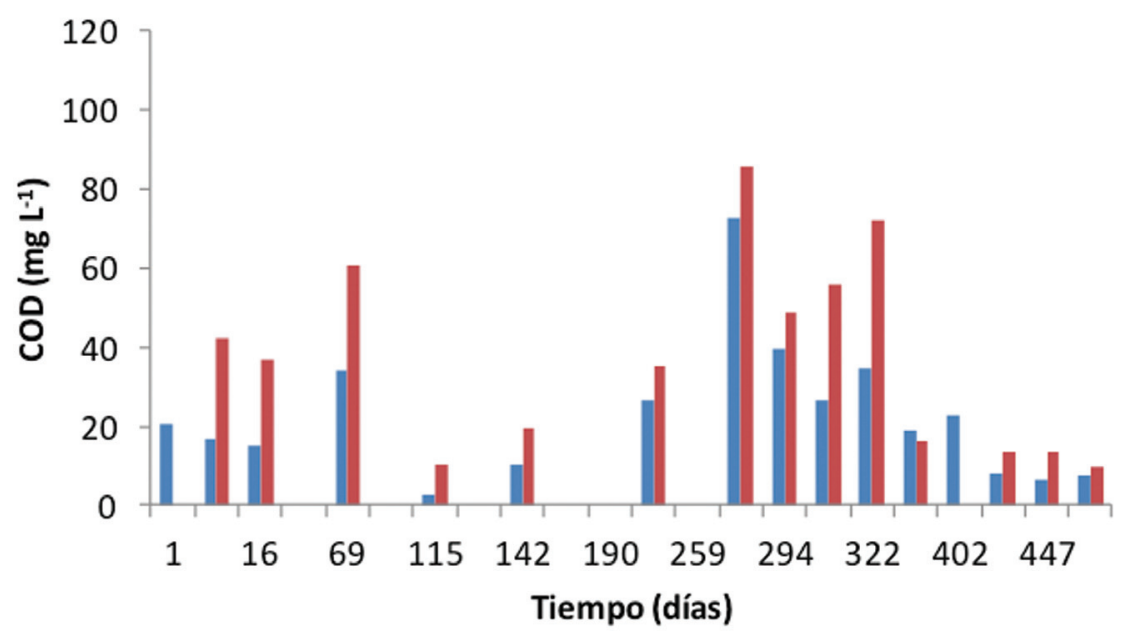

2. Escorrentía cortical

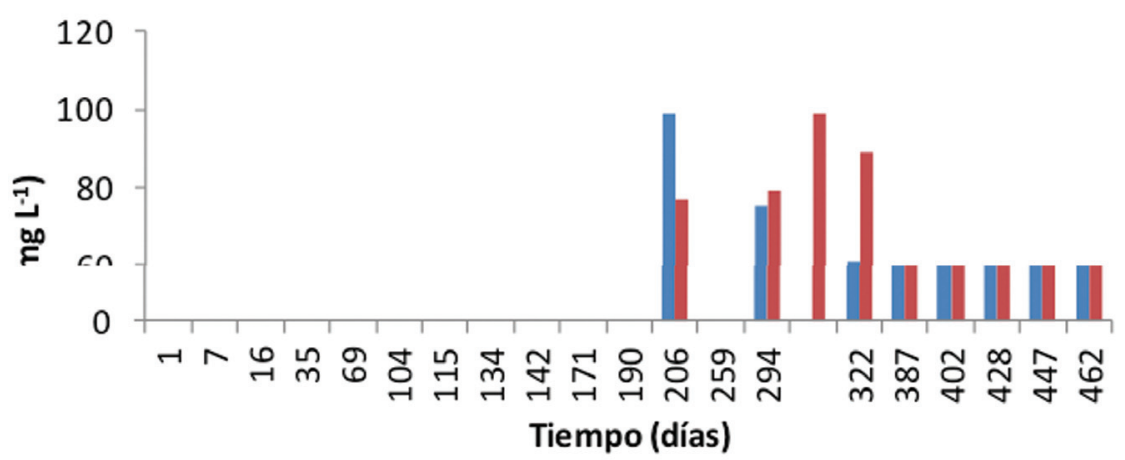

3. Trascolación

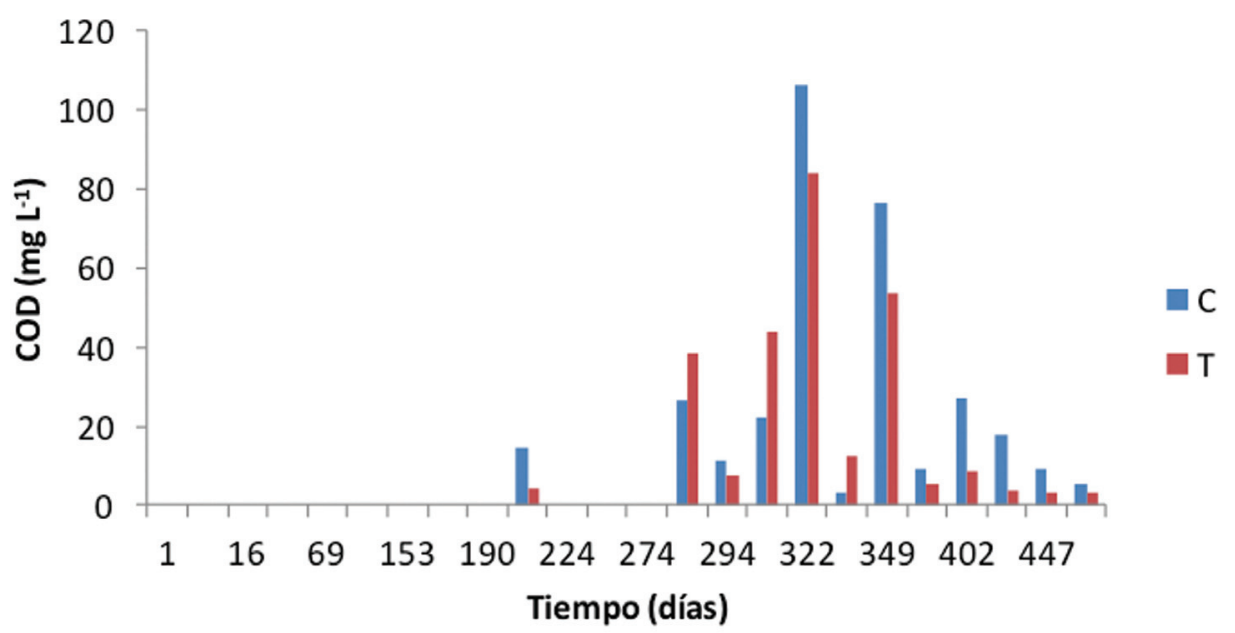

Figura 2. Dinámica de la concentración de carbono orgánico disuelto (COD) en los flujos de escorrentía en el suelo (1), escorrentía cortical (2) y trascolación (3). 
también existe una correlación significativa con el amonio. En cambio, en el flujo de trascolación sólo se correlaciona significativamente con el catión potasio.

Tabla 4. Coeficientes de correlación de Pearson entre la cantidad de agua, la absorbancia en ultravioleta y las concentraciones de nutrientes disueltos en agua analizadas por separado en función del origen del agua: escorrentía $(\mathrm{R})$, escorrentía cortical $(\mathrm{S})$ y trascolación $(\mathrm{T})(* * *, * *, y *$ indican correlaciones estadísticamente significativas al nivel del $0.001,0.01$ y 0.05 , respectivamente).

\begin{tabular}{|c|c|c|c|c|c|c|c|c|c|}
\hline & Origen & COD & Abs UV & POD & $\mathrm{P}-\mathrm{PO}_{4}$ & NOD & $\mathrm{N}-\mathrm{NO}_{3}$ & $\mathrm{~N}-\mathrm{NH}_{4}$ & K \\
\hline \multirow[t]{3}{*}{ Cantidad } & $\mathrm{R}$ & -0.264 & -0.099 & -0.157 & -0.100 & -0.335 & -0.265 & -0.033 & -0.247 \\
\hline & S & $-0.627 *$ & -0.432 & -0.111 & -0.267 & -0.144 & -0.172 & -0.187 & -0.336 \\
\hline & $\mathrm{T}$ & -0.396 & -0.104 & -0.197 & -0.172 & -0.186 & -0.150 & 0.333 & -0.236 \\
\hline \multirow[t]{3}{*}{$\mathrm{COD}$} & $\mathrm{R}$ & & $0.955 * * *$ & $0.445^{*}$ & 0.398 & $0.759 * * *$ & -0.132 & 0.360 & 0.315 \\
\hline & $\mathrm{S}$ & & $0.806^{* * *}$ & 0.482 & 0.472 & 0.125 & 0.489 & 0.264 & $0.540^{*}$ \\
\hline & $\mathrm{T}$ & & $0.612 * *$ & $0.588^{* *}$ & 0.240 & $0.661 * * *$ & $0.509^{*}$ & -0.120 & $0.690 * * *$ \\
\hline Abs UV & $\mathrm{R}$ & & & $0.397^{*}$ & 0.329 & $0.668 * * *$ & -0.234 & 0.348 & $0.380 *$ \\
\hline $\mathrm{S}$ & & & & 0.009 & $0.884 * * *$ & $0.683 * *$ & $0.908 * * *$ & $0.791 * * *$ & $0.908 * * *$ \\
\hline $\mathrm{T}$ & & & & 0.284 & $0.388^{*}$ & $0.547 * * *$ & 0.125 & 0.248 & $0.750 * * *$ \\
\hline POD & $\mathrm{R}$ & & & & $0.718 * * *$ & 0.029 & 0.172 & $0.804 * * *$ & 0.140 \\
\hline $\mathrm{S}$ & & & & & 0.031 & 0.270 & $-0.79 * * *$ & $-0.694 * *$ & 0.145 \\
\hline $\mathrm{T}$ & & & & & 0.021 & $0.572 * * *$ & $0.456^{* *}$ & 0.016 & $0.517 * * *$ \\
\hline $\mathrm{P}_{-} \mathrm{PO}_{4}$ & $\mathrm{R}$ & & & & & -0.084 & 0.363 & $0.894 * * *$ & -0.064 \\
\hline $\mathrm{S}$ & & & & & & 0.265 & $0.550^{*}$ & $0.533 *$ & $0.885^{* * * *}$ \\
\hline $\mathrm{T}$ & & & & & & 0.163 & -0.149 & -0.064 & $0.368^{*}$ \\
\hline NOD & $\mathrm{R}$ & & & & & & -0.178 & -0.042 & $0.532 * *$ \\
\hline $\mathrm{S}$ & & & & & & & $-0.532 *$ & $-0.606^{* *}$ & 0.358 \\
\hline $\mathrm{T}$ & & & & & & & $0.607 * * *$ & 0.150 & $0.764 * * *$ \\
\hline $\mathrm{N}-\mathrm{NO}_{3}$ & $\mathrm{R}$ & & & & & & & 0.310 & -0.019 \\
\hline $\mathrm{S}$ & & & & & & & & $0.984 * * *$ & 0.431 \\
\hline $\mathrm{T}$ & & & & & & & & 0.212 & $0.448 * *$ \\
\hline $\mathrm{N}-\mathrm{NH}_{4}$ & $\mathrm{R}$ & & & & & & & & 0.007 \\
\hline $\mathrm{S}$ & & & & & & & & & 0.402 \\
\hline $\mathrm{T}$ & & & & & & & & & $0.350^{*}$ \\
\hline
\end{tabular}

\subsection{Absorbancia en el ultravioleta}

La absorbancia específica en el rango de longitud de onda del ultravioleta, expresada por unidad de COD, se utiliza como una estimación del grado de aromaticidad y degradabilidad de la materia orgánica disuelta (Chantigny et al. 2008). La absorbancia en el rango del ultravioleta (Tab. 3) difiere significativamente entre los distintos flujos de agua siendo del orden de 6 a 8 veces superior en la escorrentía cortical respecto al agua de trascolación. El agua que escurre sobre la corteza disuelve 
compuestos fenólicos y por ello presenta un alto grado de aromaticidad. La absorbancia en el ultravioleta en la escorrentía cortical es ligeramente más alta en la parcela control aunque las diferencias no son significativas debido a la gran variabilidad temporal. En la parcela control, donde no se han eliminado ramas de los árboles, el recorrido es más largo y se produce mayor disolución de compuestos aromáticos. Cuando se compara el COD con la absorbancia en el ultravioleta, la pendiente proporciona la absorbancia específica por unidad de C orgánico disuelto (Fig. 3). Las pendientes difieren significativamente entre las muestras en función de su origen, siendo la pendiente en muestras de agua procedentes de escorrentía superficial y subsuperficial el doble de la escorrentía cortical y unas siete veces superior a la del flujo de agua que atraviesa la cubierta vegetal. Estos datos sugieren que los compuestos aromáticos disueltos por el agua que recorre la corteza se concentran en el agua del suelo debido posiblemente a su estabilidad frente a la degradación.

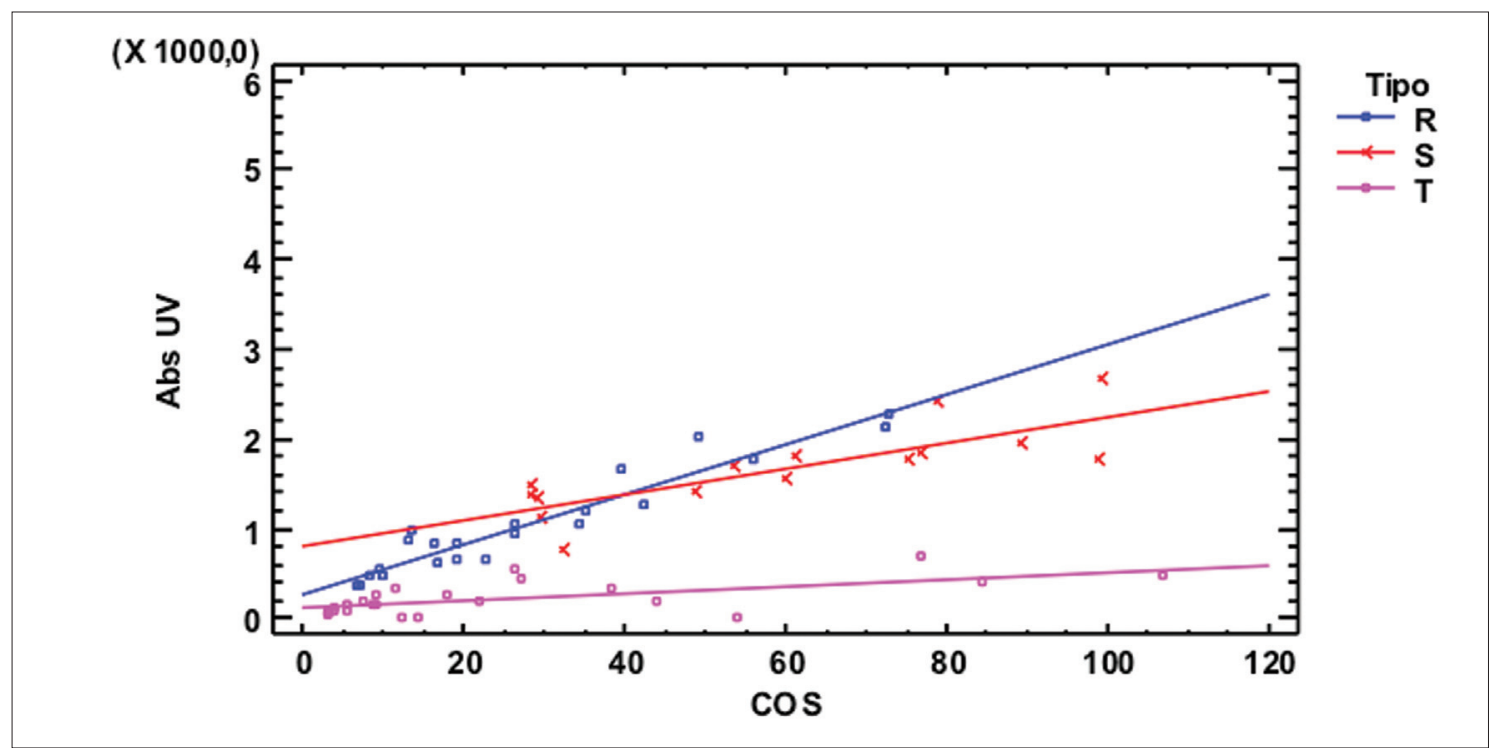

Figura 3. Comparación de rectas de regresión de absorbancia en ultravioleta vs el carbono orgánico soluble en muestras de agua procedentes de escorrentía superficial y subsuperficial en el suelo (R), escorrentía cortical (S) y trascolación (T). R-cuadrado (ajustado por g.1) $=92.38 \%$.

\section{Conclusiones}

El tratamiento selvícola no presenta a corto plazo efectos muy significativos sobre la concentración de nutrientes en el agua que fluye a través del ecosistema. El principal efecto que tiene el tratamiento se produce sobre el flujo de trascolación; el tratamiento selvícola aumenta el volumen de agua que atraviesa el dosel arbóreo y disminuye las concentraciones de nutrientes disueltos, especialmente las de las formas orgánicas disueltas.

La absorbancia en el ultravioleta expresada por unidad de carbono orgánico di- 
suelto es muy alta en las muestras precedentes de escorrentía cortical y aumenta de forma significativa en el agua que fluye a través del suelo, indicando que se produce un efecto concentración de los compuestos aromáticos.

\section{Agradecimientos}

Este trabajo se ha realizado en el marco del proyecto de investigación CGL201128776-C02-02 financiado por el Ministerio de Economía y Competitividad.

\section{Bibliografía}

Ávila, A., Piñol, J., Rodà, F. y Neal, C., 1992. Storm solute behaviour in a montane Mediterranean Forested catchment. J Hydrol. 140:143-161.

Bernal, S. Butturini, A., Sabater, F., 2005. Seasonal variations of dissolved nitrogen and DOC:DON ratios in a intermittent Mediterranean stream. Biogeochemistry 75, 351-372.

Bormann, F.H., Likens, G.E., 1979. Pattern and process in a forested ecosystem. SpringerVerlag, New York.

Chantigny, M.H., Angers, D.A., Kaiser K., Kalbitz, K., 2008. Extraction and characterization of dissolved organic matter. In: Carter, M.R., Gregorich, E.G. (eds.), Soil sampling and methods of analysis. Second edition. Taylor \& Francis group. Boca Raton FL.

Chapin, F.S. Matson, P.A., Mooney, H.A., 2002. Principles of terrestrial ecosystem ecology. Springer, Nueva York. USA.

Kimmins, J.P., 1997. Forest ecology. A foundation for sustainable management. PrenticeHall, Nueva Jersey. USA.

Kuo, S., 1996. Phosphorus. In: Spark, D. (ed.), Methods of soil analysis: Chemical methods, Part 3. Madison: American Society of Agronomy, pp. 869-919.

Lado-Monserrat L., Lull C., Bautista I., Lidón A., Herrera, R., 2014. Soil moisture increment as a controlling variable of the "Birch effect". Interactions with the pre-wetting soil moisture and litter addition. Plant Soil 379, 21-34.

Likens, G.E., Bormann, F.H., 1995. Biogeochemistry of a forested ecosystem. Springer-Verlag. New York.

Llorens, P., Domingo, F., 2007. Rainfall partitioning by vegetation under Mediterranean conditions. A review of studies in Europe. Journal of Hydrology, 335(1-2): 37-54.

Patton, C.J., Kryskalla, J. R., 2003. Methods of Analysis by the U.S. Geological Survey National Water Quality Laboratory_Evaluation of Alkaline Persulfate Digestion as an Alternative to Kjeldahl Digestion for Determination of Total and Dissolved Nitrogen and Phosphorus in Water U.S. Geological Survey. Water-Resources Investigations Report 03-4174.

Perakis, S., Hedin, L., 2002. Nitrogen loss from unpolluted South American forest mainly via dissolved organic compounds. Nature 415, 416-419.

Qualls, R.G., Haines, B.L., 1991. Geochemistry of dissolved organic nutrients in water percolating through a forest ecosystem. Soil Sci. Am. J. 55, 1112-1123.

Qualls, R.G., Haines, B.L, Swank, W.T., Tyler, S.W., 2000. Soluble organic and inorganic nu- 
trient fluxes in clearcut and mature deciduous forest. Soil Sci. Am. J. 64, 1068-1077.

Qualls, R.G., Haines, B.L., 1992. Bio degradability of dissolved organic nutrients in water percolating through a forest throughfall, soil-solution, and stream water. Soil Sci. Am. J. 56, 578-586.

Sardans, J., Rodà, F., Peñuelas, J., 2004. Phosphorus limitation and competitive capacities of Pinus halepensis and Quercus ilex subsp. rotundifolia on different soils. Plant Ecol. 174, 307-319.

Tecator Ltd UK. Application Note AN 5226. Determination of ammonium in 2M KCl soil extracts by FIAstar 5000.

Tecator Ltd UK. Application Note AN 5201. Determination of the sum of nitrate and nitrite in water by FIAstar 5000 .

Thomas, H., Stoddart, J.L., 1980. Leaf senescence. Annu. Rev. Plant Physiol. 31, 83-111.

Vanderbilt, K., Lajtha, K., Swanson, F.J., 2003. Biogeochemistry of unpolluted forested watersheds in the Oregon Cascades: temporal patterns of precipitation and stream nitrogen fluxes. Biogeochemistry 62, 87-117.

Vitousek, P.M., Gerrish, G., Turner, D.R., Walker, L.R., Mueller-Dumbois, D., 1995. Litterfall and nutrient cycling in four Hawaiian montane rainforest. J Trop Ecol 11, 189-203.

Yaalon, D.H., 1997. Soils in the Mediterranean region: What makes them different? Catena 28, 157-169.

Yakovchenko, V.P., Sikora, L.J., 1998. Modified dichromate method for determining low concentrations of extractable organic carbon in soil. Commun. Soil Sci. Plant Anal., 29, 421-433. 